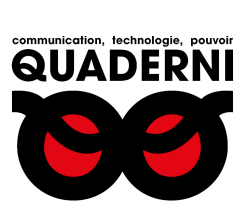

\title{
Quaderni
}

Communication, technologies, pouvoir

De l'alterscience au conspirationisme : l'exemple de la diffusion et de la réception du "documentaire » La Révélation des pyramides sur l'internet

\section{Damien Karbovnik}

\section{(2) OpenEdition \\ 12 Journals \\ Édition électronique \\ URL : https://journals.openedition.org/quaderni/1115 \\ DOI : 10.4000/quaderni. 1115 \\ ISSN : 2105-2956 \\ Éditeur \\ Les éditions de la Maison des sciences de l'Homme}

Édition imprimée

Date de publication : 5 octobre 2017

Pagination : 63-74

Référence électronique

Damien Karbovnik, « De l'alterscience au conspirationisme : l'exemple de la diffusion et de la réception du "documentaire » La Révélation des pyramides sur l'internet », Quaderni [En ligne], 94 | Automne 2017, mis en ligne le 05 octobre 2019, consulté le 22 juillet 2021. URL : http://journals.openedition.org/ quaderni/1115; DOI : https://doi.org/10.4000/quaderni.1115 


\section{Politique}

\section{De l'alterscience au}

conspirationisme : l'exemple de la diffusion et de la réception $\mathrm{du}$ "documentaire») La Révélation des pyramides sur l'internet

\section{Damien \\ Karbovnik}

Doctorant en sociologie Université Paul Valéry Montpellier III IRSA-CRI
Un internaute un peu distrait ne penserait jamais que La Révélation des pyramides ${ }^{l}$ puisse être considéré comme un produit réellement conspirationniste. Sorti officiellement en 2010, mais diffusé à grande échelle sur le net à partir de $2012^{2}$, ce film revendique le statut de documentaire et propose de l'archéologie traditionnelle une vision alternative. S'il est toujours possible d'y voir un simple discours contestataire de la science officielle - ou une simple manifestation de plus de ce que d'aucuns ont appelé la « pyramidiologie »-, c'est néanmoins faire fi du discours qui structure l'argumentation du film autant que de l'accueil plus que bienveillant qu'il reçut dans le monde francophone.

Car ce film, qui aurait été visionné sur YouTube ${ }^{3}$ plusieurs millions de fois, a fait l'objet, parmi ses détracteurs, ses défenseurs et ses concepteurs, de maints débats - par l'intermédiaire des réseaux sociaux et de « commentaires » laissés sur les pages internet parlant de lui ${ }^{4}$; et ces échanges présentent de nombreux argumentaires qui remettent en question l'exactitude des faits exposés et permettent de classer définitivement La Révélation des pyramides dans le domaine pseudo-scientifique.

Nous estimons néanmoins intéressant de passer outre l'exactitude des faits et de tâcher plutôt d'éclairer l'idéologie générale du film, de son auteur et de ses suites. Autrement dit, dans cet article, nous ne reviendrons pas sur les «faits » avancés. Nous souhaitons ne nous intéresser qu'à l'histoire générale qui y est racontée, à la manière dont elle l'est, à ce à quoi elle sert et à la mise en scène dans laquelle elle est présentée. Nous verrons ainsi que le phénomène de La Révélation 
des pyramides est profondément lié à l'espace d'expression offert par l'internet, en même temps qu'il est un élément, peut-être un peu atypique - quoique, foncièrement, assez commun -, de la « complosphère ». Parallèlement à cela, nous évoquerons la dynamique commerciale que prend le phénomène au fil de son développement.

\section{D'un documentaire à une révélation}

Doit-on, au sujet de La Révélation des pyramides, parler de film ou de documentaire ? Car à travers cette question, c'est la valeur fictionnelle même que nous interrogeons. Le film prétend-il faire œuvre de science ou assume-t-il consciemment une part libre d'imagination? Pour être en mesure de répondre à cette question, il faut mettre en balance les ambitions déclarées et ce qui en résulte, et opérer une première distinction dans la conception même du film. Y coexistent en effet deux discours simultanés : le premier est un argumentaire technique - et, a priori, scientifique -, le second, un récit étiologique, cosmologique et eschatologique.

Nous laisserons de côté le premier discours; il a déjà subi le feu de bien assez de contre-expertises ${ }^{5}$. Contentons-nous de rappeler qu'il vise à démontrer que la pyramide de Khéops n'a pu être édifiée que grâce à des connaissances inconnues de l'homme, tout comme de nombreuses autres constructions visibles en Amérique du Sud, sur l'Île de Pâques, en Jordanie, au Pakistan - et en maints autres endroits, dont l'histoire aurait perdu la trace ${ }^{6}-$, qui seraient toutes alignées le long d'un « équateur penché ».

Cette démonstration vise à préparer le terrain et à donner par avance du crédit au second discours, qui repose sur le postulat que ces sites seraient les vestiges d'une mystérieuse « civilisation des bâtisseurs », détentrice d'un savoir très supérieur au nôtre. Ainsi certaines pyramides seraient-elles des méta-machines qui contiendraient une partie de leur savoir codé 7 . Le film prétend, grâce à un informateur secret, révéler partiellement le message laissé par cette ancienne civilisation : l'apocalypse serait imminente ${ }^{8}$.

De plus, l'inversion, imminente, des pôles magnétiques terrestres sera à l'origine de la destruction de la surface de la Terre par les rayons du soleil. Mais ce n'est pas tout : on apprend aussi que les " prêtres » seraient les détenteurs d'un ancien savoir antérieur à une précédente destruction de la planète. Ce savoir proviendrait de cette fameuse civilisation des bâtisseurs qui auraient justement construit les pyramides et, à travers le globe, quantité d'autres édifices tout aussi énigmatiques, afin d'y cacher un message destiné à nous mettre en garde contre des événements cycliques qui se produiraient tous les 10000 ou 12000 ans et seraient capables d'anéantir l'humanité.

Le film soutient alors la thèse fondamentale que les bouleversements climatiques que nous pouvons actuellement observer avertiraient de l'imminence du prochain cataclysme et qu'il est urgent de s'y préparer. Pour cela, il est impératif de mener de vraies recherches historiques et archéologiques pour comprendre l'intégralité du savoir laissé par cette ancienne civilisation, car si elle nous a prévenu de notre proche disparition, elle a pu aussi nous laisser des indications pour nous aider à y échapper. 
En présentant les choses ainsi, on comprend sans difficulté l'ambivalence du film, et si nous avons évacué toutes les démonstrations « scientifiques », on n'en fait que mieux ressortir un des biais cognitifs essentiels du film : il procède à une révélation sur un plan tant eschatologique qu'historique, puisque - tout en nous prévenant de notre possible prochaine disparition - il nous révèle aussi que l'histoire et l'archéologie, telles qu'elles sont habituellement pratiquées et enseignées, sont inexactes.

\section{Une rhétorique alterscientifique et conspira- tionniste}

Maintenant qu'après cette brève présentation du film, son indigence scientifique apparaît clairement, ajoutons encore quelques éléments qui feront mieux ressortir sa rhétorique conspirationniste, et revenons en particulier sur ce mystérieux informateur.

Réalisé par Patrice Pooyard, le film présente toutes les apparences du documentaire : une narratrice, Alika Del Sol, raconte l'histoire en voix-off, parfois interrompue par des interviews d' « experts ». Mais ce qu'on doit relever avant tout est le caractère énigmatique de la source des idées avancées : curieusement, elle n'est jamais précisée par la narratrice, qui la garde secrète et se contente de l'attribuer à « un chercheur hors normes, infatigable et rigoureux ${ }^{10} »$

Si mystérieux qu'il soit, cet informateur, qui se dévoilera lui-même assez rapidement après la diffusion du film sur internet, est pourtant cité dès le début. Le générique du film mentionne en effet « D’après La Révélation des pyramides de
Jacques Grimault $»^{11}$. Ce point est important, car il permet de mettre en évidence la construction du film. Si la narratrice n'est que narratrice, la relation qui lie le réalisateur Pooyard à la source Grimault est en revanche circulaire: l'un et l'autre n'ont de cesse de rappeler, en interview, que le film ne reflète que la démarche qu'a entreprise Pooyard, à titre personnel, à partir des travaux de Grimault ${ }^{12}$. Toutefois, un problème demeure, puisque La Révélation des pyramides n'existe pas en tant que livre ${ }^{13}$. De plus, comme le révèle le générique de fin, le texte du film est bien une œuvre commune à eux deux ${ }^{14}$.

Quoi qu'il en soit, cette précision permet de comprendre que le véritable idéologue du film est bien Grimault, personnage mystérieux dont on ne connaît pas les qualifications ${ }^{15}$, qu'il refuse d'indiquer ${ }^{16}$, mais qui s'octroie volontiers des compétences en biologie, physique, linguistique, histoire et archéologie ${ }^{17}$ : Grimault, comme il le déclare volontiers en interview, affirme faire de la science ${ }^{18}$.

Mais, à bien écouter et regarder le film, on se rend compte que, même pour de la vulgarisation scientifique, le ton est dissonant : présenté « comme la plus grande chasse au trésor de l'histoire ${ }^{19} »$ et comme «l'enquête qui change le monde ${ }^{20}$ », il constitue du début à la fin une violente charge contre l'archéologie et l'histoire officielles. Et si la rhétorique conspirationniste y est discrète, elle n'y en demeure pas moins bien présente, car dès lors qu'on explique que l'égyptologie est entachée d'un dogmatisme aveugle, en prétendant qu'elle va jusqu'à empêcher de véritables recherches, on suggère clairement l'idée qu'on nous cache sciemment des choses ${ }^{21}$. 
De plus, lorsqu'on voit avec quelle insistance on tente de nous convaincre que dans tous ces vestiges archéologiques tout est lié, grâce à cette mythique civilisation des bâtisseurs, et que rien n'est dû au hasard - puisque cette civilisation détenait un savoir aujourd'hui perdu -, on retrouve les trois règles du théoricien du complot ${ }^{22}$. Enfin, quand on se rend compte que la finalité de ce film ne vise qu'à rétablir la « vraie histoire » de notre passé, tout en nous avertissant de l'imminence de l'apocalypse et de la vacuité de la science officielle, on comprend qu' on a bel et bien affaire à un produit caractéristique de l'alterscience conspirationniste ${ }^{23}$.

\section{Une forme nouvelle pour un fonds ancien}

Si on y regarde de plus près, les idées avancées dans La Révélation des pyramides ne sont pas vraiment nouvelles. Pour comprendre le lien entre La Révélation des pyramides et l'occultisme, il suffit de savoir que, pendant dix-sept ans, Grimault fut membre actif de l'Association Atlantis, y assumant presque toutes les responsabilités possibles, de celle de conférencier à celle de président ${ }^{24}$. Fondée en 1926 par Paul Le Cour, cette association a pour but «l'étude des traditions, en vue de rechercher ce que toutes les civilisations ont appelé la Tradition primordiale, pour permettre une rénovation spirituelle de l'humanité $»^{25}$ et publie une revue du même nom, avec pour sous-titre Archéologie scientifique et traditionnelle. De fait, on peut voir dans $L a$ Révélation des pyramides la concrétisation des travaux menés par l'association depuis plusieurs décennies, agencés et structurés par Grimault.

De plus, il convient également d'intégrer Grimault et son discours dans une tradition littéraire plus récente, et tout aussi éclairante ${ }^{26}$. C'est en 1960 , avec la parution du Matin des magiciens ${ }^{27}$, ouvrage coécrit par Louis Pauwels et Jacques Bergier, que naît un phénomène littéraire sans réel précédent, porté jusqu'en 1971 par la revue Planète. Mêlant sans états d'âme ésotérisme, occultisme, histoire, archéologie, science et science-fiction, les deux auteurs innovent dans un genre particulier, le réalisme fantastique, et posent les bases d'un véritable paradigme alternatif à celui de la « science officielle », qu'ils souhaitent voir explorer par d'autres chercheurs. Une multitude d'auteurs à succès s'inscriront dans cette mouvance, de Robert Charroux à Graham Hancock, en passant par Erich von Däniken ou Zacheria Sitchin, tous autodidactes, tous cités par Grimault en interview ${ }^{28}$. Dans ce «nouveau » paradigme, on valorise la science avec conscience : à la science officielle, qui a abouti à la bombe atomique par manque d'éthique, on oppose l'alchimie, dans la mesure où elle serait l'archétype de la science vertueuse. Dans le même temps est développée une nouvelle voie d'accès à la connaissance, qui combine sciences, ésotérisme et imagination. La connaissance ainsi obtenue devrait être absolue, et être la seule capable de retirer l'Occident de l'impasse dans laquelle il se serait engagé, et qui le menacerait d'une disparition imminente.

Dans ce paradigme, on s'intéresse au paranormal, à l'ufologie et à la parapsychologie, dans la mesure où ces disciplines sont susceptibles de nous offrir une meilleure compréhension de notre réalité ${ }^{29}$. Mais, plus que tout, ce qui forme l'ossature de ce paradigme est la théorie des Ancêtres Supérieurs. Cette théorie postule 
que, dans un passé lointain, la Terre aurait vu s'épanouir des civilisations qui, depuis lors, à la suite de cataclysmes d'origines naturelles ou humaines, auraient disparu. Et si nous parlons bien de la théorie des ancêtres supérieurs - et non pas des Anciens Astronautes, comme c'est habituellement le cas -, la raison en est assez simple : dans l'ouvrage fondateur, n'est indiquée pour ces civilisations avancées aucune origine. De fait, dans les publications ultérieures de ce genre, on trouve différentes variantes.

En somme, La Révélation des pyramides n'est qu'un nouvel avatar contemporain de ces productions caractéristiques des années 1960-1970. Toutefois, si ce qui change est avant tout le mode choisi - celui du film - et le moyen de diffusion - l'internet -, il faut aussi reconnaître que le ton conspirationniste est, lui aussi, relativement nouveau.

Bien qu'Olivier Dard remarque dans cette mouvance réaliste fantastique ${ }^{30}$ la présence d'un discours conspirationniste, nous pensons qu'elle n'est qu'un développement tardif. Certes, toutes les composantes se trouvent réunies dès Le Matin des magiciens, mais ce n'est qu'au fil des années que la rhétorique conspirationniste gagne en importance jusqu'à devenir centrale dans la plupart des productions actuelles apparentées.

\section{Un élément atypique de la « complosphère »}

Quand on observe la réception sur l'internet de La Révélation des pyramides, il ressort que ce qui dans le documentaire a le plus séduit n'est pas tant la théorie globale - celle de la civilisation des bâtisseurs -, que l'idée qu'on nous cache notre véritable histoire. Ainsi, en commentaire de la vidéo sur YouTube ${ }^{31}$, on peut lire ceci :

" Abi pedicatum: Je ne dis pas que cette enquête nous donne la vérité mais le problème c'est qu'elle n'en donne pas moins que l'archéologie traditionnelle... »

"Sara Sara : superbe documentaire!!! c'est comme pour l'histoire des religions et l'histoire tout court il y aura toujours des gens pour venir nous demander de s'en tenir aux histoires officielles alors qu'ils n'étaient même pas présents...»

"Christian : Excellent mais la plus grande vérité est ailleurs... [...] Les Pyramides sont des émetteurs de communication à $30^{\circ}$ en direction de Nibiru. [...]»

"Tesla: Tu n'as pas conscience de tout ce que l'on vous cache, ça dépasse de loin la construction de ces pyramides (qui ne servaient qu'à aider nos ancêtres, beau cadeau de la part des Orionides). Nous vivons dans un système d'exploitation des masses à tous les niveaux de la société (pharmaceutiques, technologie, monétaires, scientifiques...), à l'échelle planétaire depuis plus de 50 ans. »

On observe ainsi un profond basculement. Dans son étude sur la théorie des anciens astronautes, Wiktor Stoczkowski soulignait qu'arguments et preuves étaient tout à fait secondaires, et que seule importait la théorie globale ${ }^{32}$. À travers $L a$ Révélation des pyramides, on se rend compte que seules comptent les preuves, la théorie globale allant parfois jusqu'à être oubliée. Qu'importe ce 
que les preuves démontrent ou permettent de démontrer, pourvu qu'elles contredisent la science officielle : point de vue qui se retrouve chez bon nombre de partisans du film, mais surtout chez Grimault. Ce dernier tient un discours circulaire, qui prend parfois des tournures assez radicales et illustre de flagrantes lacunes méthodologiques ${ }^{33}$.

Bien qu'il prétende « faire de la science », il ne cite aucune autre source que son propre ouvrage - que n'a lu personne d'autre que Pooyard - et justifie l'intégralité de sa démarche par sa capacité personnelle à raisonner comme le faisaient les «Anciens », c'est-à-dire les bâtisseurs. Autrement dit, Grimault est le seul à faire de la vraie science, parce qu'il est le seul à en connaître les vraies méthodes, bien qu'il ne les explique jamais. On comprend donc qu'étant plus ou moins autodidacte, il a pour seule méthode son intuition ${ }^{34}$.

Cette attitude est lourde de conséquences, car elle le pousse à rejeter en les dénigrant les scientifiques officiels, qu'ils soient égyptologues ou biologistes, au seul motif que, selon lui, ces derniers ne raisonnent pas correctement ${ }^{35}$ - opinion qui le conduit à défendre des personnalités pour le moins fort éloignées du ton général du film ${ }^{36}$. C'est ainsi qu'on le voit défendre Robert Faurisson, « un type extrêmement courageux ${ }^{37}$ », dont il reprend notamment la version négationniste de l'histoire du Journal d'Anne Frank ${ }^{38}$ et, dans un autre registre, on peut aussi l'entendre parler occasionnellement d'Illuminati ${ }^{39}$. Tout aussi surprenante peut paraître sa proximité avec la « fachosphère », par l'intermédiaire d'Égalité \& Réconciliation et d'un cycle de conférences qui, en 2015, passa notamment par le Théâtre de la Main d'or.
Nous voilà bien loin de la civilisation des bâtisseurs, mais tout cela illustre particulièrement bien ce qui le relie à la « complosphère » : l'histoire est trafiquée de manière à asservir l'humanité, mais lui-même est là pour rétablir la vérité et nous aider à découvrir par qui, comment et pourquoi ${ }^{40}$. Dès lors, ce glissement vers l'extrême-droite apparaît-il aussi comme un changement de stratégie de communication et la tentative d'exploiter un nouveau marché potentiel qui partagerait une forme de pensée commune, bien qu'a priori fort éloigné de la cible première.

Très présent sur internet, grâce à ses propres vidéos et à ses nombreuses interventions dans des médias alternatifs du web, comme dans l'émission Bob vous dit Toute La Véritét ${ }^{41}$, ou encore sur MetaTV, il bénéficie dans le monde francophone d'une diffusion sans précédent ${ }^{42}$.

\section{Champ de recherche ou business plan?}

Cette visibilité est d'autant plus surprenante que Grimault n'apporte pas d'éléments réellement nouveaux par rapport à son film. Il se contente d'en répéter les idées essentielles, en se campant dans une attitude dogmatique et radicale qui vise à insulter sans ménagement ceux qui ne pensent pas comme lui, sans jamais tenir le moindre discours proprement scientifique ${ }^{43}$. En fait, sa stratégie de communication est extrêmement simple et ne repose aucunement sur d'éventuelles démonstrations. Dès qu'on lui reproche des imprécisions ou qu'on lui demande plus de détails, il renvoie invariablement soit à son livre ${ }^{44}$, qui n'est pas publié, soit à de prochains films qui seraient prévus ${ }^{45}$, soit encore à l'association qu'il a créée après avoir quitté Atlantis en 2010, La Nouvelle Atlantide ${ }^{46}$. 
L'ensemble de ces développements du film initial peut se justifier d'un point de vue émique par deux moyens. D'une part, La Révélation des pyramides se veut un enseignement et, dans cette optique, Grimault insiste sur le fait qu'il fait œuvre de pédagogie et de didactique ${ }^{47}$ : ainsi peuton considérer toutes ses initiatives postérieures - livres, films, association - comme des prolongements destinés à permettre aux intéressés de comprendre petit à petit l'intégralité de la vérité dans toutes ses dimensions. Mais en même temps, cette démarche prétend aussi familiariser les chercheurs au mode de pensée des «Anciens ».

D'un point de vue étique, cela ressemble à s'y méprendre à un business plan bien rôdé. Expliquons-nous : Grimault utilise son livre non publié pour justifier tous ses calculs. En somme, il crée pour son livre ce que Hans-Robert Jauss appelle un « horizon d'attente $»^{48}$. Certes, il affirme qu'il ne peut publier son livre en raison de problèmes juridiques ${ }^{49}$, mais il n'en demeure pas moins qu'il crée surtout, chez le public, une attente.

S'il fonde La Nouvelle Atlantide, association qui a pour but de promouvoir « le Bon, le Vrai, le Juste et le Beau $\|^{50}$, c'est aussi un excellent moyen de diffuser ses publications, d'après lui au nombre de $70^{51}$, ainsi que l'éphémère revue Nouvelle Planète - aucune ressemblance avec le projet initial de Pauwels et Bergier ne semble fortuite -, et de donner à ses conférences ${ }^{52}$ une assise, rien de tout cela n'étant, bien entendu, gratuit.

Pour finir, évoquons la suite de La Révélation des pyramides, qui est annoncée depuis plusieurs années ${ }^{53}$. Différée pour des problèmes juridiques et des tensions entre les deux principaux scéna- ristes, Grimault et Pooyard , elle a fait l'objet de différents projets, mais tous menés grâce au crowdfunding. Si le projet dirigé par Pooyard, intitulé L'Enquête continue, a réussi à mobiliser beaucoup de monde ${ }^{54}$ et devrait, après plusieurs reports, voir enfin le jour en septembre 2017, la tentative menée par Grimault, de son côté, semble avoir plus de mal et peine à réunir le financement demandés5.

Mais, plus que le procédé du crowdfunding, c'est le principe des « contreparties » qui doit être remarqué. Pour illustrer notre propos, nous ne prendrons pour exemple que le projet mené par Pooyard et la société Jayan films. Ainsi, dans L'Équateur penché - titre de travail de L'Enquête continue -, contre $2 €$, on reçoit le lien de visionnage en streaming du prochain film en avantpremière ; contre $5 €$, on bénéficie en plus de cela du droit de recevoir la newsletter du projet, et on est ainsi tenu régulièrement au courant de l'avancement de la réalisation du film; contre $10 €$, on a accès de surcroît à la musique du film. Plus les sommes augmentent, plus importantes sont les contreparties : elles comprennent différents types de gratifications y compris symboliques, allant de livres sur la réalisation du film à la mention de son nom dans le générique, en passant par des entretiens privilégiés avec les réalisateurs du film ou encore des tee-shirts de soutien.

Bien que non exhaustive, cette liste permet de comprendre que la suite de La Révélation des pyramides n'est pas qu'un film ni la simple quête d'une vérité : bien entendu, si ces contreparties sont censées n'être pour les donateurs que des facteurs de motivation, il n'en demeure pas moins qu'elles constituent un réel business parallèle au 
film. Et, dans ce cadre précis, il n'est rien proposé de moins, en termes wébériens, qu'une forme de bien de salut. En effet, les contreparties apparaissent comme des biens symboliques, gérés par un groupe de personnes spécialisé et destinés à satisfaire les attentes existentielles de leur public.

\section{L'internet, espace d'expression parallèle}

Après ce bref exposé, il apparaît donc indubitable qu'on ne saurait considérer ni La Révélation des pyramides ni tout ce qui s'ensuivit comme des productions caractéristiques de la science. En revanche, il est plus juste de considérer ce phénomène comme la résurgence d'un discours plus ancien, adaptée aux conditions techniques et technologiques de notre époque : on ne publie plus de livres, on réalise des films; on ne diffuse plus dans des librairies, on diffuse directement sur l'internet. Mais cette adaptation s'exerce aussi sur certains points du discours, et la dimension conspirationniste en est une conséquence immédiate.

Il est très tentant de comprendre le développement de la thèse conspirationniste comme une conséquence de l'incapacité du discours alterscientifique à s'imposer. En effet, si les thèses défendues par La Révélation des pyramides ne sont pas prises au sérieux par la science officielle, la raison est qu'il doit exister une conjuration qui empêche la vérité de s'exprimer. Si l'on nous cache la vérité, ce n'est que pour mieux nous contrôler.

En pleine ère post-factuelle, un tel film tente en avançant des faits de s'imposer comme un produit scientifique, mais lorsqu'on prête réellement attention à son argumentation, on ne trouve pas le moindre fait : rien d'autre que des interprétations, nécessairement subjectives et à charge. Donc aucune probabilité que les théories du film soient validées par la communauté scientifique, et c'est la raison pour laquelle l'internet devient un média idoine. Il n'est en effet besoin d'aucune validité scientifique pour pouvoir y être publié, ni même reconnu. Cette manière de s'intéresser au grand public sans aucun intermédiaire et sans passer par la validation des pairs est caractéristique de l'alterscience ${ }^{56}$.

Cependant, le premier film n'est qu'un appât : il ne révèle pas tout et prétend ne dévoiler qu'une infime partie de la vérité. Dès lors suscite-t-il interrogations et attentes sur lesquelles il est possible de capitaliser, ce que précisément vont faire Grimault et Pooyard en multipliant les interviews dans des médias alternatifs. Ils vont certes là où ils ont la possibilité de s'exprimer librement, mais surtout là où ils trouvent aussi celle de s'adresser à un auditoire réceptif, qui ralliera d'autant plus facilement leurs thèses qu'il appartient plus ou moins ouvertement à la « complosphère ». C'est aussi dans cet esprit qu'il est possible de comprendre le rapprochement entre Grimault et Égalité \& Réconciliation : s'il peut exister quelque proximité idéologique entre ce dernier et l'association, par contre le film et l'association n'ont rien d'autre en commun qu'une concordance d'atmosphère conspirationniste.

La constitution de forums et de groupes Facebook permet d'observer la formation d'une communauté de fans qui s'apparentent aux seekers ${ }^{57}$; mais elle va surtout contribuer au développement du phénomène, qui pourra ainsi faire valoir 
l'argument du nombre.

Cette attitude de seeker est absolument fondamentale, car elle détermine les possibilités d'accroissement du phénomène. Face à des fans en attente d'éléments nouveaux et d'informations toujours plus sensationnelles, il est possible de proposer toute une panoplie de produits susceptibles de répondre à ces attentes, des plus simples aux plus prestigieuses. Ainsi peut-on simplement visionner les films ultérieurs, se former pour arriver à « raisonner comme les Anciens » ou ni plus ni moins que se voir inscrire sur une sorte de liste de « mécènes », honorant ceux qui, par leurs dons particulièrement généreux, auront rendu possible la suite de la révélation.

Mais s'il est une chose qui ne doit pas nous échapper, c'est bien que le contenu du film est profondément conspirationniste. La manière dont est monté et présenté le récit tend à faire croire qu'il ne s'agit que d'une recherche scientifique. Pour notre part, nous avons souvent eu tendance à n'y voir qu'une forme de quête initiatique. Mais l'essentiel du discours n'est pas dans le récit en soi, ni dans son aboutissement; il est dans sa valeur contestataire du discours scientifique officiel et les ressorts sur lesquels il s'appuie. Il est un énième moyen de démontrer que l'histoire est sciemment altérée par certains, et que Grimault et Pooyard ne sont jamais que de nouvelles figures de prophètes de l'ère dite de la post-vérité.

Ainsi le phénomène de La Révélation des pyramides témoigne-t-il du développement d'une variante singulière de pensée conspirationniste, parallèle à la science et aux médias officiels. Sans l'internet et les réseaux de la « complosphère », il paraît peu probable qu'un tel phénomène puisse se développer de nos jours. Cependant, grâce à une rhétorique bien particulière et une habile stratégie de communication, le phénomène conquiert de nouveaux marchés, s'autofinance et se pérennise, tout en offrant à ses partisans de véritables biens de salut, propres à signifier leur appartenance au phénomène. 
$\mathrm{N} \cdot \mathrm{O} \cdot \mathrm{T} \cdot \mathrm{E} \cdot \mathrm{S}$

1. Le documentaire fut mis en ligne, puis retiré à plusieurs reprises : www.youtube.com/watch? $\mathrm{v}=$ HFpHDXUgcqw. Toutes les adresses internet données dans cet article ont été vérifiées valides à la date du 07/05/2017.

2. Signalons néanmoins que le film a pu bénéficier de diffusions sur la chaîne RMC Découverte, notamment le $02 / 01 / 2017$.

3. On trouve régulièrement sur internet la mention de plus de 17 millions de vues dès la première mise en ligne sur You Tube. Malheureusement, le documentaire fut supprimé à de nombreuses reprises, puis remis en ligne par différentes personnes. Il est aujourd'hui impossible de chiffrer réellement le nombre de vues de ce documentaire.

4. Par exemple, citons la critique fortement humoristique de Tsuki et remarquons surtout les échanges dans les commentaires : http://www. nioutaik.fr/index.php/2013/02/26/640-la-revelationdes-pyramides-le-documentaire-en-mousse.

5. Citons par exemple un site dédié tout spécifiquement à démonter le film : https:// imposturedejacquesgrimault.wordpress.com.

6. www.youtube.com/watch?v=HFpHDXUgcqw, à partir de $1 \mathrm{~h} 13 \mathrm{~min}$.

7. www.youtube.com/watch? $\mathrm{v}=\mathrm{A} \operatorname{soEY} 18 \mathrm{gv} 1$, à partir de 40min20s.

8. www.youtube.com/watch? $\mathrm{v}=\mathrm{HFpHDXUgcqw}$, à partir de $1 \mathrm{~h} 36 \mathrm{~min}$.

9.www.youtube.com/watch? $\mathrm{v}=\mathrm{HFpHDXUgcqw \& t=}$ $4425 \mathrm{~s}, 12 \mathrm{~min} 47 \mathrm{~s}$.

10. www.youtube.comwatch? $\mathrm{v}=\mathrm{HFpHDXUgcqw} \& \mathrm{t}=$ $4425 \mathrm{~s}, 1 \mathrm{~min} 55 \mathrm{~s}$.

11. Par exemple : www.youtube.com/watch? $\mathrm{v}=$ AsoEY18gv1w, à partir de 2min39s.
12. Il est toutefois à noter que le livre paraît finalement en autoédition en 2017. D'abord vendu en souscription, il est à présent disponible sur le site des Éditions de La Nouvelle Atlantide (http://editionslna.org/). Remarquons aussi qu'il porte la mention « Tome 1 » et se voit présenté comme l'ouvrage qui a inspiré le film, alors qu'il a été publié plusieurs années après sa sortie.

13. www.youtube.com/watch? $v=H F p H D X U$ gcqw\&t $=4425 \mathrm{~s}, 1 \mathrm{~h} 39 \mathrm{~min} 15 \mathrm{~s}$.

14. On ne dispose d'aucunes informations biographiques concrètes sur Grimault. Après avoir travaillé 22 ans dans le tourisme, il se consacrerait depuis 1993 à ses recherches personnelles et à leur valorisation, par le biais de publications, de conférences et de cours, notamment au sein de l'association Atlantis. Comme il l'affirme lui-même dans ses interviews, il fut initié à la «culture des anciens " par son grand-père, " simple campagnard » (https://www.baglis.tv/intervenants/2138-jacquesgrimault.html).

15. www.youtube.com/watch?v=OL0E3zpAB_A, $8 \min 52 \mathrm{~s}$.

16. www.youtube.com/watch? $v=0 L 0 E 3 z p A B \_A$, à partir de 1 h07min.

17. www.youtube.com/watch?v=OL0E3zpAB_A, à partir de 1 h05min.

18. www.youtube.com/watch? $v=H F p H D X U g c q w \& t$ $=4425 \mathrm{~s}, 1 \min 16 \mathrm{~s}$.

19. www.youtube.com/watch? $v=H F p H D X U g c q w \& t$ $=4425 \mathrm{~s}, 2 \min 24 \mathrm{~s}$.

20. www.youtube.com/watch? $\mathrm{v}=\mathrm{AsoEY} 18 \mathrm{gv} 1 \mathrm{w}$, à partir de $22 \mathrm{~min} 20 \mathrm{~s}$.

21. Véronique Campion-Vincent, La Société parano, théories du complot, menaces et incertitudes, Paris, Payot, 2007 (2005), p. 10-11.

22. Alexandre Moatti, Alterscience. Postures, dogmes, idéologies, Paris, Odile Jacob, 2013. 
23. https://web.archive.org/web/20170705050030/ http://www.nouvelle-atlantide.org/association/ constitution.html.

24. www.association-atlantis.org/a-propos.

25. Pour les lignes qui vont suivre, nous nous appuyons sur un ensemble d'éléments que nous avons définis dans le cadre de notre thèse : L'ésotérisme grand public : le Réalisme Fantastique et sa réception. Contribution à une sociohistoire de l'occulture.

26. Louis Pauwels et Jacques Bergier, Le Matin des magiciens, Paris, Gallimard, 1960.

27. www.youtube.com/watch? $\mathrm{v}=\mathrm{AsoEY} 18 \mathrm{gv} 1 \mathrm{w}$, à partir de $2 \mathrm{~h} 05 \mathrm{~min}, 2 \mathrm{~h} 49 \mathrm{~min}$ et $3 \mathrm{~h} 16 \mathrm{~min}$.

28. Il suffit de faire une brève recherche des conférences données par Grimault et mise à disposition sur You Tube pour retrouver les mêmes centres d'intérêts.

29. Olivier Dard, « Le complot, moteur de l'histoire dite "secrète" », in Raison Publique, n¹6, Juin 2012, p. 67-77.

30. www.youtube.com/watch? $\mathrm{v}=$ HFpHDXUgcqw

31. Wiktor Stoczkowski, Des hommes, des dieux et des extraterrestres, ethnologie d'une croyance moderne, Paris, Flammarion, 1999.

32. Cet aspect du discours de Grimault a été mis en évidence au cours de son interview à La Tronche en Live, une émission zététicienne diffusée sur Radio Campus Lorraine : https://www.youtube.com/ watch? $v=O L 0 E 3 z p A B \_A$.

33. Il défend notamment l'idée d'une école qui développerait " l'intuition du vrai » https://www. youtube.com/watch? $=$ AsoEY18gv1w, à partir de $1 \mathrm{~h} 13 \mathrm{~min}$.

34. Il prend, entre autres, l'exemple de l'universitaire Didier Kahn qui a certes passé 25 ans à étudier l'alchimie, mais n'y aurait rien compris (https:// www.youtube.com/watch? $\mathrm{v}=$ AsoEY18gv1w, à partir de 2 h56min).
35. Si le lien entre le négationnisme, l'antisémitisme et La Révélation des pyramides est tout sauf évident, il faut tout de même remarquer que les prises des positions de Grimault sur ces sujets sont pour le moins ambiguës. Ainsi peut-on l'entendre expliquer, au cours de son interview pour MetaTV, pendant près de dix minutes, que les Hébreux «n'ont pas d'histoire », qu'ils « trafiquent l'histoire » et qu'ils « s'attribuent des mérites qu'ils n'ont pas, des choses qu'ils auraient faites qu'ils n'ont pas faites ». https:// www.youtube.com/watch? $v=$ AsoEY18gv1w, à partir de 3 h00 min.

36. www.youtube.com/watch? $\mathrm{v}=$ AsoEY18gv1w, $37 \mathrm{~min} 20 \mathrm{~s}$.

37. www.youtube.com/watch? $\mathrm{v}=\mathrm{AsoEY} 18 \mathrm{gv} 1 \mathrm{w}$, à partir de 1 h35min.

38. www.youtube.com/watch? $=$ AsoEY18gv1w, à partir de $2 \mathrm{~h} 23 \mathrm{~min}$.

39. www.youtube.com/watch? $=A \operatorname{AsoEY} 18 \mathrm{gv} 1 \mathrm{w}$, à partir de $1 \mathrm{~h} 33 \mathrm{~min}, 1 \mathrm{~h} 55 \mathrm{~min}, 3 \mathrm{~h} 03 \mathrm{~min}$.

40. Par exemple : www.youtube.com/watch?v=D_ hKSNv8kyM. Cette interview du 8 avril 2013 est le quatrième passage des auteurs du film dans l'émission.

41. Bien que La Révélation des pyramides ait bénéficié de doublages et de sous-titrages en plusieurs langues, le film n'a pas reçu d'accueil aussi important à l'étranger, notamment en raison de l'existence de nombreux produits anglophones concurrents.

42. Son passage à l'émission Tronche en Live illustre parfaitement cet aspect.

43. Par exemple : www.youtube.com $/$ watch? $v=$ AsoEY18gv1w, à partir de $3 \min 30$.

44. Par exemple : www.youtube.com $/$ watch? $\mathrm{v}=$ AsoEY18gv1w, 1h45min.

45. http://www.nouvelle-atlantide.org/.

46. www.youtube.com/watch? $\mathrm{v}=\mathrm{AsoEY} 18 \mathrm{gv} 1 \mathrm{w}$, $39 \mathrm{~min}$. 
47. Hans-Robert Jauss, Pour une esthétique de la réception, Paris, Gallimard, coll. « tel », 2013 (1990), pp. 54-58.

48. Comme l'explique l'émission Tronche en Live, cet argument est peu convaincant, mais il renforce l'idée d'un complot contre l'auteur.

49. http://www.nouvelle-atlantide.org/.

50. www.youtube.com/watch?v=AsoEY18gv1w, à partir de $1 \mathrm{~h} 22 \mathrm{~min}$.

51. http://www.nouvelle-atlantide.org/activites/ conferences.html

52. En attendant la sortie de la suite du premier film, un autre film, intitulé Gizeh 2005, est sorti en 2015 afin d'approfondir le premier, dont de ce fait il ne constitue pas à proprement parler la suite.

53. Si nous mettons en avant dans cet article la rupture entre Grimault et Pooyard, il faut tout de même préciser que le film initial était porté par trois personnes. Outre les deux déjà citées, il convient d'ajouter Olivier Krasker-Rosen, dont le nom apparaît dans le générique de fin de La Révélation des pyramides, pour l'associer à l'écriture du scénario. Ce passionné d'alchimie, qui est le premier à avoir pris ses distances avec les deux autres, est réellement apparu dans le débat lorsque Grimault dénonça ses agissements comme étant susceptibles de nuire à la réalisation de la suite du film. De fait, après avoir déposé une marque au nom de «La Révélation des pyramides », Krasker-Rosen rendit impossible toute suite sans son accord. La bataille fit rage sur l'internet entre les trois protagonistes pendant des années, sans trouver d'issue réelle, sinon celle de l'abandon du projet unique et commun au profit de démarches personnelles.

54. https://fr.ulule.com/lequateur-penche-deuxiemeetape/.

55. www.helloasso.com/associations/la-nouvelleatlantide/collectes/la-revelation-des-pyramides-2-1- equateur-penche.

56. Alexandre Moatti, op. cit., pp. 47-48.

57. Dans sa définition du cultic milieu, sorte de marché underground d'idées religieuses, politiques, scientifiques et culturelles, le sociologue Colin Campbell caractérise le comportement des personnes qui y prospectent par le terme de seekers. Ces personnes ne se contentent pas d'écouter ; elles sont aussi en recherche permanente et mettent à l'épreuve les croyances qu'on leur propose, de sorte à établir leur propre système de croyances. Colin Campbell, « The Cult, the Cultic Milieu and Secularization », in A sociological Yearbook of Religion in Britain, $\mathrm{n}^{\circ} 5$, 1972, pp. 119-136. 